\title{
Wi-Fi Indoor Positioning Fingerprint Health Analysis for a Large Scale Deployment
}

\author{
KS Yeo ${ }^{* 1}$, A Ting ${ }^{\# 2}, \mathrm{SC} \mathrm{Ng}^{\# 3}$, D Chieng ${ }^{\# 4}$, N Anas ${ }^{\# 5}$ \\ *Department of Electrical and Electronics Engineering, Faculty of Engineering and Technology, Tunku Abdul Rahman University College, \\ Jalan Genting Kelang, Setapak, 53300 Kuala Lumpur \\ Email:yeoks@tarc.edu.my \\ ${ }^{\#}$ Wireless Innovation, MIMOS Berhad, Technology Park Malaysia, Bukit Jalil, \\ 57000 Kuala Lumpur \\ Email: ${ }^{\# 2}$ kee.ting@mimos.my; ${ }^{\# 3}$ sc.ng@mimos.my; ${ }^{\# 4}$ ht.chieng@mimos.my; ${ }^{\# 5}$ nuzli.anas@mimos.my
}

\begin{abstract}
Indoor positioning systems (IPS) have witnessed continuous improvements over the years. However, large-scale commercial deployments remain elusive due to various factors such as high deployment cost and lack market drivers. Among the state of the art indoor positioning approaches, the Wi-Fi fingerprinting technique, in particular, is gaining much attention due to its ease of deployment. This is largely due to widespread deployment of WiFi infrastructure and its availability in all existing mobile devices. Although WiFi fingerprinting approach is relatively low cost and fast to deploy, the accuracy of the system tends to deteriorate over time due to WiFi access points (APs) being removed and shifted. In this paper, we carried out a study on such deterioration, which we refer to as fingerprint health analysis on a 2 million square feet shopping mall in South of Kuala Lumpur, Malaysia. We focus our study on APs removal using the actual data collected from the premise. The study reveals the following findings: 1) based on per location pin analysis, $\sim \mathbf{5 0 \%}$ of APs belong to the mall operator which is a preferred group of APs for fingerprinting. For some location, however, the number of operator-managed APs are too few for fingerprinting positioning approach. 2) To maintain mean error distance of $\sim 5$ meters, up to $80 \%$ of the APs can be removed using the selected positioning algorithms at some locations. At some other locations, however, the accuracy will exceed $5 \mathrm{~m}$ upon $>20 \%$ of APs being removed. 3) On average, around $40 \%-60 \%$ of the APs can be removed randomly in order to maintain the accuracy of $\sim 5 \mathrm{~m}$.
\end{abstract}

Keywords - indoor location positioning; fingerprint; Wi-Fi

\section{INTRODUCTION}

The transportation industry has witnessed an unprecedented disruption brought by location-based services such as Grab and Uber. Despite the success, the usage is only limited to outdoor as GPS signal is not able to penetrate through building structures. Realizing the gap, efforts have intensified in the development of an indoor positioning system in recent years. Its social and commercial values are estimated to worth around USD10 billion by 2020 [1]. Among the crucial areas that can benefit from indoor positioning systems include public safety (e.g., E911 emergency call, in building rescue), retail (e.g., store search in a shopping complex, mobile advertising, asset tracking), special care group (e.g., children and special disabled needs), etc. [2].

Various techniques have been proposed to build an indoor positioning system such as those based on Wi-Fi signals [3][5], Bluetooth signals [6], [7], FM radio signals [8], [9], RFID signals [10]-[13], sound waves [14], [15], light signals
[16], [17] and magnetic field [18], [19]. Among these techniques, the Wi-Fi fingerprinting approach is gaining much attention due to two main reasons. Firstly Wi-Fi access points (APs) are widely available across most commercial and residential premises. This enables indoor location positioning system to be deployed rapidly with minimal cost. Secondly, Wi-Fi module is available in almost every consumer device such as a smartphone, tablet, laptop, and wearable. This serves as a great commercial advantage as there is no need to provide extra hardware to the mass users [20].

The deployment of a $\mathrm{Wi}-\mathrm{Fi}$ fingerprinting indoor positioning system involves two phases: an offline phase (fingerprint calibration) and online phase (real-time positioning) [21]. During the offline phase, a site survey is conducted to record all APs within the scanning range and their associated received signal strength indicator (RSSI) at every reference point (location pin) of interest. With these records, the fingerprint database can then be constructed. During the online phase, a mobile device continuously scans 
its nearby Wi-Fi signals (online fingerprint) and initiates a query to the Wi-Fi fingerprint database through a positioning algorithm. The current location of the mobile device is determined by the algorithm which found the closest match between the online fingerprint and the fingerprint in the offline database. Inevitably, the positioning accuracy will be adversely affected if the online fingerprint on the site changes, e.g., due to the removal or relocation of Wi-Fi APs.

Besides, the desired online fingerprint can also be affected by the changes in interior partitions, walls, beams, and so on. When this happens, the offline fingerprint database needs to be updated accordingly. However, site survey and fingerprinting re-calibration work are time-consuming and labor-intensive. It is therefore important to carry out a fingerprint health analysis to investigate the degree of the mismatch between online and offline fingerprint that will yield unacceptable positioning performance.

In this work, we have deployed a large scale indoor positioning system based on the Wi-Fi fingerprinting technique in a shopping complex located at the south of Kuala Lumpur, Malaysia. Three well-known positioning algorithms used in fingerprinting approach namely Correlation, Bayesian and Weighted-K Nearest Neighbor (WKNN) were adopted to compute the users' locations. In order to evaluate the robustness of the algorithms, the online Wi-Fi fingerprint health is deliberately deteriorated, and its impact on error distance is analyzed. The primary challenge in deploying indoor positioning system in public places is that the real-time or online Wi-Fi fingerprint is highly dynamic as it changes over time, typically in weeks or months [22].

Several studies had been carried out to investigate the impact of Wi-Fi fingerprint changes on the performance of indoor positioning. In [23], Wi-Fi fingerprint was gathered from two multi-storey buildings and a shopping mall, with over a thousand of APs on the sites. In order to identify the most significant APs that impacted on positioning accuracy, a group of APs was deliberately removed (based on criteria such as APs group under highest RSSI value, entropy value, and dissimilarities measures) from the offline fingerprint database before performing positioning using Bayesian and Weighted-Centroid algorithms. Such removal of APs simulates the condition when APs are "turned off" or "missing."

Results show that about $50 \%$ APs can be safely removed from the training database while still maintaining a decent positioning accuracy. In a separate study by Esia et al. [24], there are redundant or "useless" Wi-Fi fingerprint data which do not contributing positively to the positioning processes, in which they can be determined by the total number of distinct RSSI values, percentage of missing RSSI values and overall standard deviation of the fingerprint dataset. This useless Wi-Fi fingerprint can be removed from the offline database for the benefit of reduced computational time as well as computational power without significant compromise on the positioning accuracy.

Another factor that can alter the existing radio map, besides "missing APs," is through APs movement. To deal with this situation, [22] proposed a Localization with Altered APs and Fingerprint Updating (LAAFU) system to identify any altered APs and filter them out before initiating positioning estimation. Once the positioning is done, the fingerprint of the client will be collected to update the database. This automatic fingerprint updating process is known as implicit crowdsourcing, which significantly reduces the need of extra site survey. Field studies show $20 \%$ improvement in positioning error when compared with traditional schemes. An inner-city localization system using Wi-Fi fingerprint method has been demonstrated [25], [26]. The test field covers $25 \mathrm{~km} 2$ in size which consist of private residences, office buildings, and shopping sites with approximately 60,000 APs discovered. This 15-month study concludes that the localization accuracy remains acceptable until $50 \%$ of the APs have disappeared, and a yearly site survey is recommended to resist $\mathrm{Wi}-\mathrm{Fi}$ infrastructure changes.

This paper aims to compare and contrast our findings with the existing studies. The studies above differ regarding algorithm implementations, experiment methodologies, and actual environments. By selecting a typical shopping mall, we hope to gain valuable practical insights into whether these well-known indoor positioning algorithms are ready for mass users.

\section{MATERIAL AND METHOD}

Our test site is a 4-storey shopping mall with a size of around 1.5 million square feet. It houses more than 380 retailers, with a visiting crowd number of approximately 2 million per month. In this paper, we only present the results obtained from the ground floor which spans from the central court to the west-wing of the shopping mall (refer to Figure 1(a)).

Results from other floors are found to exhibit a similar trend. A total of 69 pins in the form of Cartesian coordinate were defined on the ground floor. The decision on where to position the pins is based on the location of the facilities provided by the mall operator in the interest of the general public e.g. retail shop's main entrance, lift entrance, restroom, ATM, etc. Due to that, the pins do not follow a uniform grid structure. In our opinion, the pin grid not only needs to be flexible enough to cater for various kind of layout designs of a particular building but also to reduce unnecessary calculation on less important locations.

Subsequently, the fingerprint data on each defined pin was collected on the actual location using an in-house Android application running on a smartphone (Brand: Xiaomi Mi 3). Each fingerprint sample data consisted of BSSID, SSID, timestamp and Received Signal Strength Indicator (RSSI) of the heard (or scanned) Wi-Fi access points (APs). For each pin, a total of 20 fingerprint data samples were collected -5 samples from each orthogonal orientation e.g. North-East-South-West, similar to the procedure described in [3]. The data samples from 4 orthogonal orientations enables a more accurate view on how fingerprint data reacts against the human body's actual orientation. The fingerprint data collection process was done during the peak business hours in order to gain insights on how the system copes with big crowd.

The pins location and the number of APs heard are summarized in Figure 1(b). 


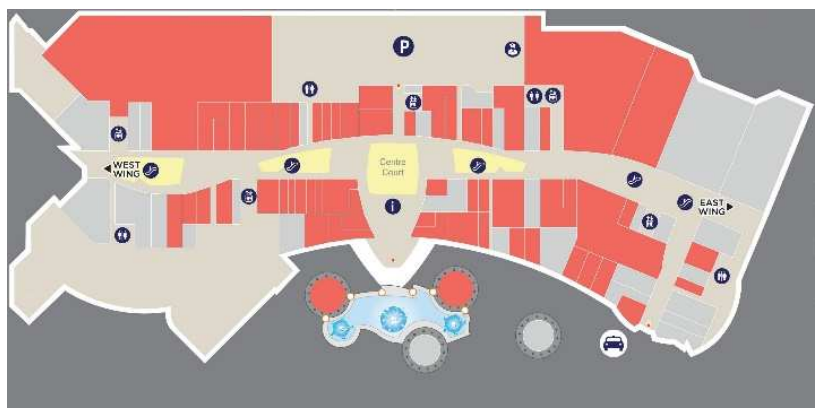

1(a)

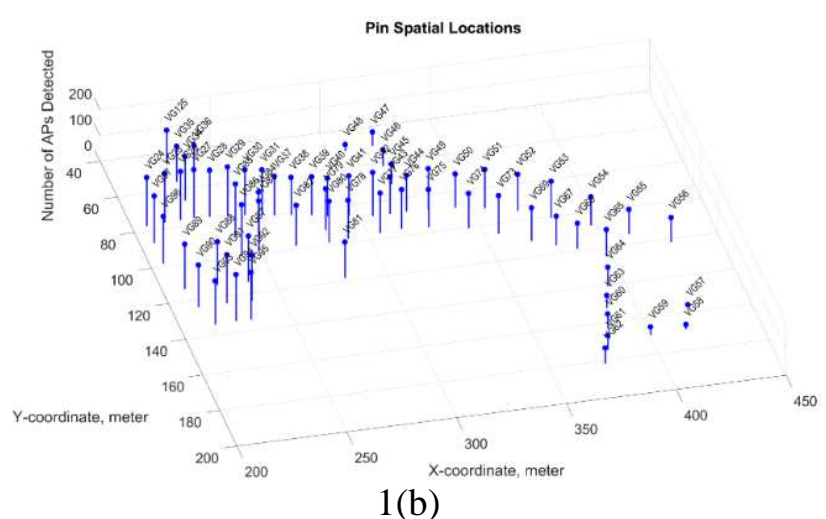

Fig. 1 (a) Ground floor layout of the shopping mall (b) Ground floor test site defined with 69 location pins

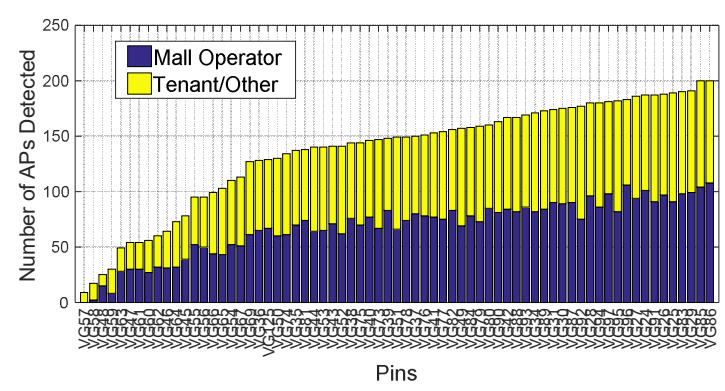

Fig. 2 Number of APs detected at each pin location. Blue bars represent the number of APs owned by the mall operator; yellow bars represent the number of tenants/other Apps

Figure 2 summarizes the number of APs heard at each pin's location, sorted in ascending order. It is not surprising to observe a big difference regarding AP density on the same floor, i.e. the lowest count of APs is at VG57 (9 APs detected) while the highest count of APs is at VG85 and VG86 (200 APs detected). As we can see, VG57 is at the far end of the West Wing while VG85 and VG86 are located at the central court of the shopping mall. Note that the central court of the shopping mall is a podium where it has a clear, direct line of sight from all levels.

Hence this location is expected to have the most number of APs heard. We have categorized the apps into two categories, namely 1) Mall Operator's APs which are deployed by the mall operator and 2) Other APs which are either set up by tenants or visitors. The categorization of the APs is possible because all the APs deployed by Mall Operator carry the same SSIDs. These APs are typically fixed to the building. The other APs which could be fixed or mobile are generally "noisy" as their properties are incredibly dynamic. For example, tenants who cease their business operation are likely to remove their APs while mobile hotspot users are subjected to random appearance. According to the data collected, the composition ratio of the Mall Operator's APs to Other APs is about 50:50.

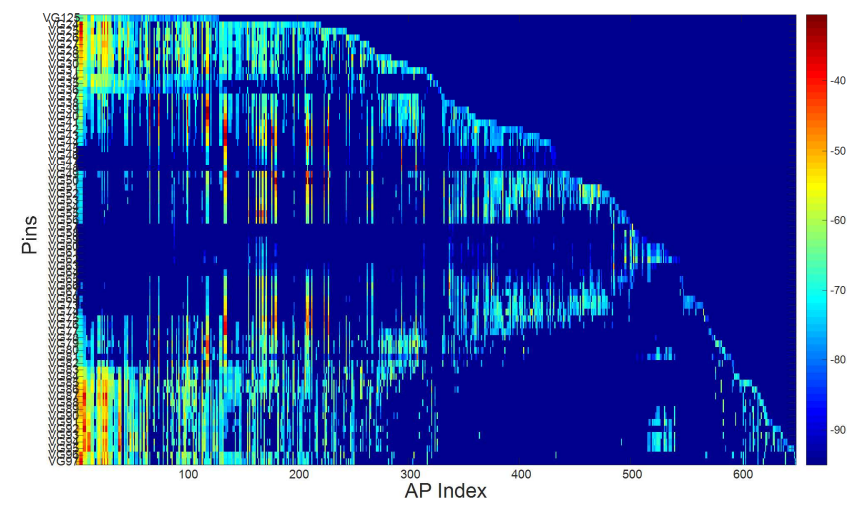

Fig. 3 Wi-Fi Radio map.

Figure 3 shows the Wi-Fi radio map of the test site. The $\mathrm{Y}$-axis consists of all the defined pins while the $\mathrm{X}$-axis consists all the APs heard at the entire test site. The color code is used to represent the intensity of the mean RSSI values in $\mathrm{dBm}$. This radio map provides a quick grasp of information. For instance, if we draw a horizontal line across any particular pin, and from there we could know which APs are heard by this pin and their associated mean RSSI. Likewise, if we draw a vertical line across an AP Index, we could know which locations (pins) that this particular AP has appeared. This information is particularly useful when comes to the study of fingerprint data fluctuation or fingerprint health evaluation, as generally, the fingerprint data of a public venue is highly dynamic [22].

\section{RESULTS AND DISCUSSION}

As mentioned earlier, three positioning algorithms which are commonly used by the fingerprint approach namely Correlation Coefficient, Bayesian and weighted k-nearest neighbor (WKNN) are put under test to estimate user's location. These algorithms are described in the following sections:

\section{A. Correlation Coefficient}

The Pearson correlation coefficient of vector A and vector $\mathrm{B}$ are defined as [27]:

$$
\rho(A, B)=\frac{1}{N-1} \sum_{i=1}^{N}\left(\overline{\frac{A_{l}-\mu_{A}}{\sigma_{A}}}\right)\left(\frac{B_{i}-\mu_{B}}{\sigma_{B}}\right)
$$

Where $\mu_{A}\left(\mu_{B}\right)$ and $\sigma_{A}\left(\sigma_{B}\right)$ are the mean and standard deviation of vector $A(B)$ respectively. In this case, the vectors $A$ is the online RSSI values and vector B is the RSSI values from the offline database. The correlation coefficient are calculated throughout all the RSSI values in the offline database, and the user location are estimated to be at the location pin with highest correlation to the value.

\section{B. Bayesian}

Using Bayes rule, the probability of getting Cartesian location $\mathrm{L}$ given measured signal y is defined as [28]: 


$$
p(L \mid y)=\frac{p(y \mid L) p(L)}{p(y)}
$$

Here the $p(y \mid L)$ is the likelihood of getting signal y given at location $\mathrm{L}, p(L)$ is the prior probability of location $\mathrm{L}$ being correct, and $p(y)$ is the normalizing constant. The prediction of user location is by selecting the highest probability $p(L \mid y)$ across all possible locations, $L_{i}$, thus

$$
\begin{aligned}
\widehat{L} & =\operatorname{argmax}_{\mathrm{Li}}\left[p\left(L_{i} \mid y\right)\right] \\
& =\operatorname{argmax}_{\mathrm{Li}}\left[\frac{p\left(y \mid L_{i}\right) p\left(L_{i}\right)}{p(y)}\right]
\end{aligned}
$$

We omit $p(y)$ from the equation because $p(y)$ is a constant. If we assume no prior location knowledge and all location are equally possible, $p\left(L_{i}\right)$ is also a constant and can be omitted from equation. Hence, the equation become

$$
\widehat{L}=\operatorname{argmax}_{\mathrm{Li}}\left[p\left(L_{i} \mid y\right)\right]=\operatorname{argmax}_{\mathrm{Li}}\left[p\left(y \mid L_{i}\right)\right]
$$

Where $p\left(y \mid L_{i}\right)=p_{v_{i}}\left(y-\bar{a}_{i j}\right)$ and $\boldsymbol{v}_{\boldsymbol{i}}=\boldsymbol{y}-\bar{a}_{i j}$, with $\bar{a}_{i j}$ being the mean RSSI value measured from APj at location i. Equation (4) also known as Maximum Likelihood Estimation (MLE) [29]. Further on, if we assume random vector $\boldsymbol{v}_{\boldsymbol{i}}$ is the the independent, then

$$
p\left(y \mid L_{i}\right)=\prod_{j=1}^{n_{y}} p_{v_{i}}\left(y_{j}-\bar{a}_{i j}\right)
$$

$p_{v_{i}}\left(y_{j}-\bar{a}_{i j}\right)$ is the Gaussian distribution of each measured RSSI at a particular location.

\section{WKNN}

In WKNN, the formula to estimate the Cartesian location $\hat{x}=(x, y)$ is given by [30]:

$$
\hat{x}=\sum_{i=1}^{K}\left(\frac{w_{i}}{\sum_{j=1}^{K} w_{j}}\right) \cdot P_{i}
$$

where $K$ are the number of nearest neighbour (reference points/pins). $w_{i}$ is non-negative given by:

$$
w_{i}=\frac{1}{\left\|\bar{y}-\bar{a}_{i}\right\|_{p}}
$$

and

$P_{i}=$ centre of cell the of $i$ th the calibration point

$\bar{y}=$ mean measured RSSI vector from several APs

$\bar{a}_{i}=$ vector of means RSSI values of each AP at $i$ ththe the reference point.

$\|\cdot\|_{p}=$ Euclidean norm of $p$.

Here in order to calculate $w_{i}$, the Euclidean norm of $\left\|\bar{y}-\bar{a}_{i}\right\|_{2}(p=2)$ would be:

$$
\left\|\bar{y}-\bar{a}_{i}\right\|_{2}=\sqrt{\sum_{j=1}^{N}\left|\bar{y}_{J}-\bar{a}_{J}\right|^{2}}
$$

where $\mathrm{N}$ is the size of the RSSI vector.

Figure 4 shows the scattered plot of error distances recorded at all pins while the APs are systematically (in the order of descending RSSI values) removed from the online phase for all three algorithms: (a) Correlation Coefficient, (b) Bayesian and (c) WKNN. From this scattered plot we can easily see that the error distance values are becoming more dispersed as the \% of APs removed increases, with Correlation Coefficient having the most sparse error distance values followed by Bayesian and WKNN. These scattered plots give us an overall insight of the robustness of the algorithm towards AP removal. It is worth pointing out that even though Bayesian and WKNN algorithm could maintain shallow error distance values $(<5$-meter error) up to $70 \%$ missing APs while Correlation Coefficient algorithm could only withstand only up to $20 \%$ missing APs, the computing time for Correlation Coefficient calculation are significantly faster than the other two. Since real-time positioning result is generally expected, the computation time is becoming a key criterion for the selection of the positioning algorithm.
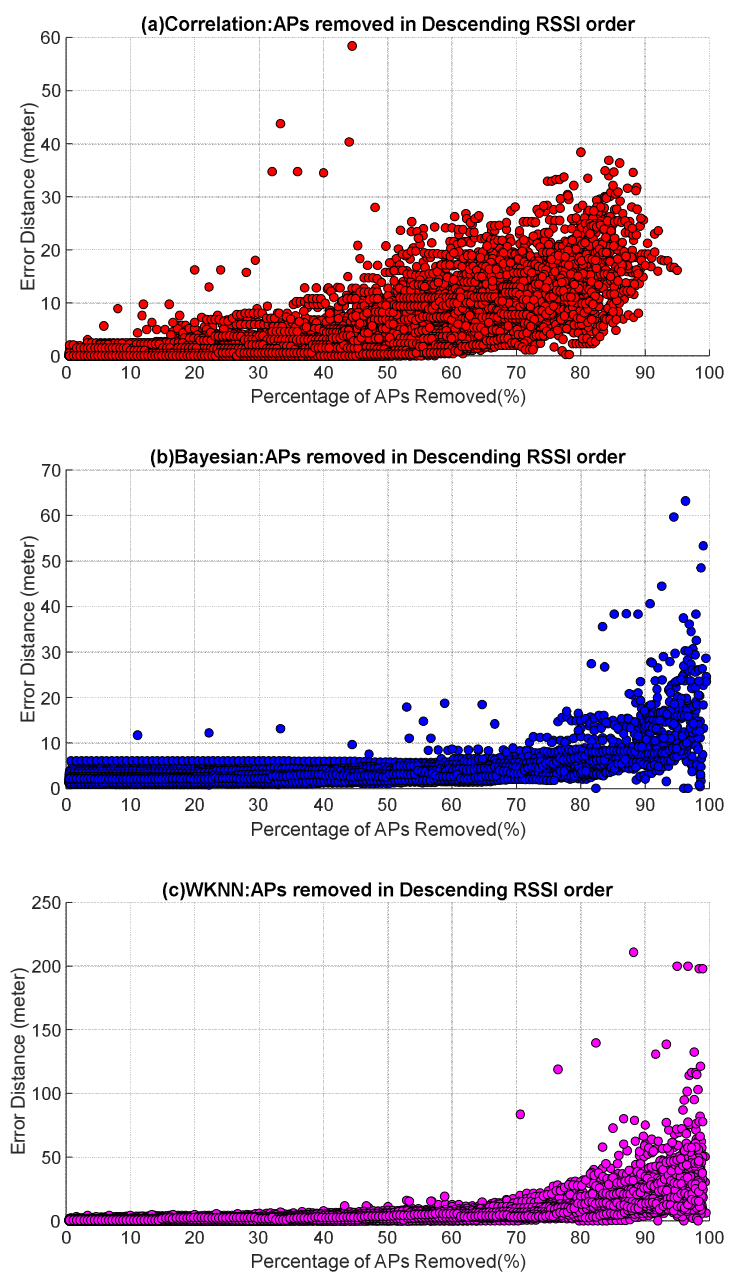

Fig. 4 Overlay of error distance calculated using (a) Correlation Coefficient (b) Bayesian (c) WKNN algorithm for all pins. 
In contrast to Figure 4, Figure 5 shows the individual pin tolerance level towards missing APs from the online phase, for (a) Correlation Coefficient (b) Bayesian and (c) WKNN algorithms. The solid lines in the graphs correspond to the percentage of APs that can be removed from the online phase while the localization accuracy on a particular pin can be maintained below 5 meters. The yellow bar indicates the total number of APs can be removed from the online phase while keeping the error distance below the threshold. In other words, a pin is more robust if 1) the dot on the solid line is high up in the graph, or 2) in another perspective the yellow bar length is much longer than the blue bar. A careful comparison on Figure 5(a), (b) and (c) reveals that the solid lines (yellow bar) are collectively higher up (longer) for the case of Bayesian and WKNN.

To further quantify the performance, the mean value of the solid line for Bayesian, WKNN, and Correlation Coefficient is $64 \%, 62 \%$, and $44 \%$ respectively. On the other hand, the highest percentage of APs could be removed from the online phase is $89 \%$ in the case of WKNN, $88 \%$ in the case of Bayesian and $77 \%$ in the case of Correlation Coefficient. The careful reader will also notice that there are missing bars in Figure 5(b). This is because the error distance calculated using the Bayesian algorithm does not reach the 5-meter threshold before the algorithm fails.
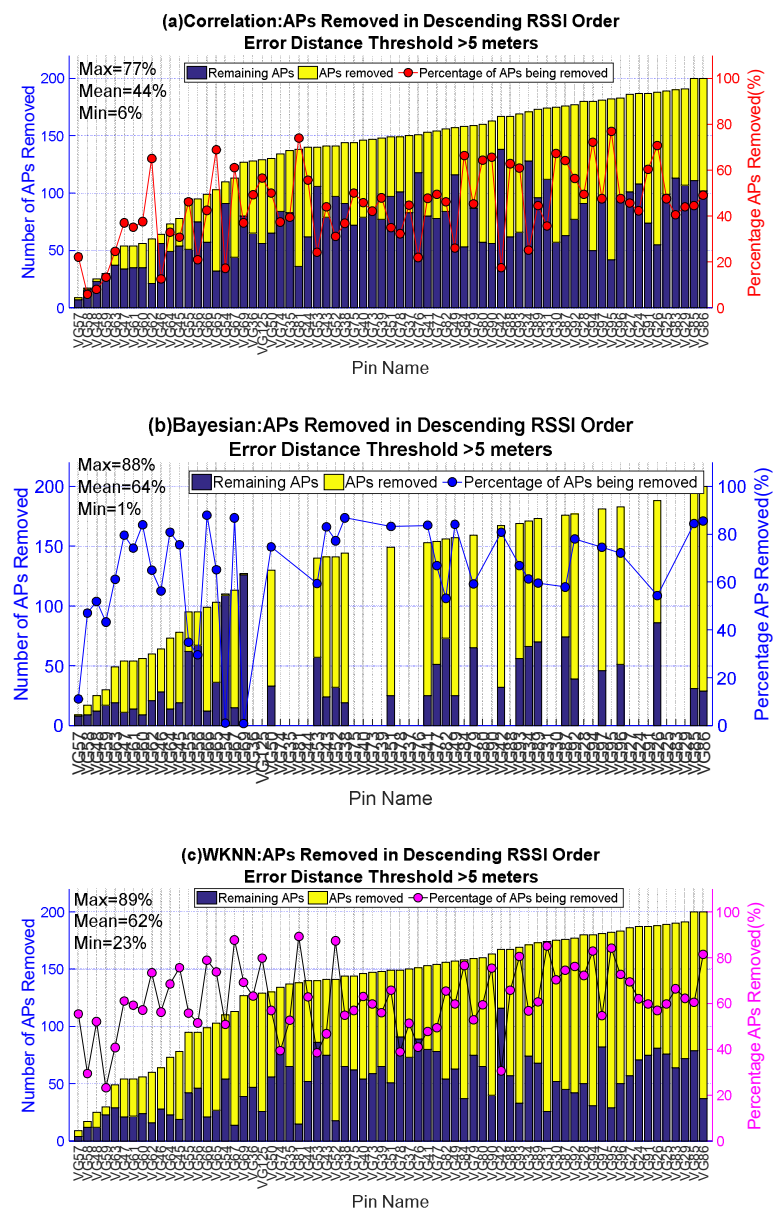

Fig. 5 Each pin can withstand a maximum number of missing APs before reaching a 5-meter error distance threshold: (a) Correlation Coefficient (b) Bayesian (c) WKNN algorithm.
Next, if we generalize that the mean percentage of missing APs from online phase at a given error distance threshold reflects the tolerance level of the positioning algorithms, then Figure 6 clearly shows that the Bayesian and WKNN algorithms outperform Correlation Coefficient algorithm. In Figure 6, the mean percentage of missing APs are evaluated at the error distance threshold between 1 to 20 meters. In general, when the error threshold range is below 4 meters, the robustness performance of all three algorithms are comparable to each other. It is only when the error distance threshold range beyond 4 meters, we can undoubtedly claim that Bayesian and WKNN algorithms can withstand higher missing APs number at any given error distance threshold as compare to Correlation Coefficient algorithm. For example, the Bayesian and WKNN algorithm could withstand $80 \%$ of (mean) missing APs at 10 meters error distance threshold requirement, while Correlation Coefficient Algorithm would easily surpass the 10 meters threshold at $60 \%$ of (mean) missing APs. Hence, it is important for the system designer to aware of the trade-off before making decision on the adoption of a particular positioning algorithms, i.e., light and fast positioning computation but with slight inaccuracy (Correlation Coefficient), or high robustness positioning algorithm but at the expense of massive computational resources (Bayesian and WKNN algorithm.

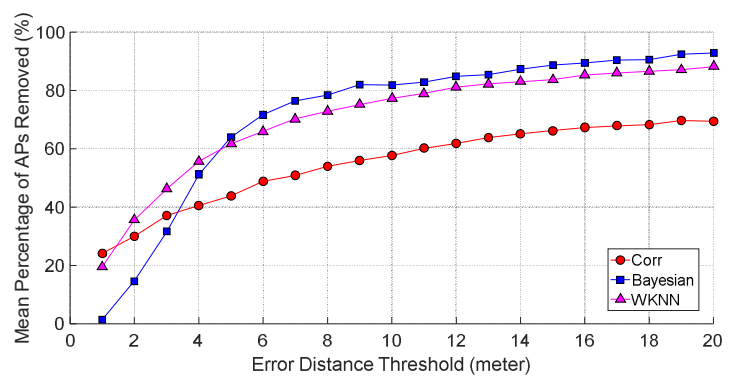

Fig. 6 Mean of missing APs (in percentage) before reaching various distance threshold: Comparison among Correlation Coefficient, Bayesian and WKNN algorithm.

\section{CONCLUSION}

In summary, we have demonstrated a large scale indoor positioning system deployment based on the Wi-Fi fingerprint technique. The Wi-Fi radio map, or also known as fingerprint database, were collected from a prominent shopping mall located at the south Kuala Lumpur, Malaysia. In order to evaluate the robustness of the indoor positioning system, the online fingerprint data has been systematically deteriorated, and subsequently compared with the offline fingerprint database using the Correlation Coefficient, Bayesian and WKNN positioning algorithms. The results show that Bayesian and WKNN appear to the most robust algorithms in mitigating missing APs scenario but at the expense of computing speed. On the other hand, the Correlation Coefficient provides a lightweight solution with satisfactory accuracy with the condition that the overall changes of Wi-Fi APs should be deliberately kept minimum.

In this paper, results are merely generated by a single type of smartphone. A further investigation on how the selected algorithms fare across different smartphone models needs to 
be carried out in order to give a fairer insight on whether these algorithms are practical for the mass market.

\section{REFERENCES}

[1] "Indoor Location in Retail: Where Is the Money? | ABI Research." [Online]. Available: https://www.abiresearch.com/marketresearch/product/1013925-indoor-location-in-retail-where-is-themon/. [Accessed: 23-Apr-2018].

[2] A. H. Sayed, A. Tarighat, and N. Khajehnouri, "Network-based wireless location: challenges faced in developing techniques for accurate wireless location information," IEEE Signal Process. Mag., vol. 22, no. 4, pp. 24-40, Jul. 2005.

[3] P. Bahl and V. N. Padmanabhan, "RADAR: an in-building RFbased user location and tracking system," in Proceedings IEEE INFOCOM 2000. Conference on Computer Communications. Nineteenth Annual Joint Conference of the IEEE Computer and Communications Societies (Cat. No.00CH37064), 2000, vol. 2, pp. $775-784$ vol.2.

[4] A. P. Rahmadini, P. Kristalina, and A. Sudarsono, "Optimization of Fingerprint Indoor Localization System for Multiple Object Tracking Based on Iterated Weighting Constant - KNN Method," Int. J. Adv. Sci. Eng. Inf. Technol., vol. 8, no. 3, pp. 998-1007, Jun. 2018.

[5] R. D. Ainul, P. Kristalina, and A. Sudarsono, "Modified Iterated Extended Kalman Filter for Mobile Cooperative Tracking System," Int. J. Adv. Sci. Eng. Inf. Technol., vol. 7, no. 3, pp. 980-992, 2017.

[6] S. Liu, Y. Jiang, and A. Striegel, "Face-to-Face Proximity EstimationUsing Bluetooth On Smartphones," IEEE Trans. Mob. Comput., vol. 13, no. 4, pp. 811-823, Apr. 2014.

[7] X. Zhao, Z. Xiao, A. Markham, N. Trigoni, and Y. Ren, "Does BTLE measure up against WiFi? A comparison of indoor location performance," in European Wireless 2014; 20th European Wireless Conference, 2014, pp. 1-6.

[8] Y. Chen, J. Liu, D. Lymberopoulos, and B. Priyantha, "FM-based Indoor Localization," Microsoft Res., Jun. 2012.

[9] S. Yoon, K. Lee, and I. Rhee, "FM-based Indoor Localization via Automatic Fingerprint DB Construction and Matching," in Proceeding of the 11th Annual International Conference on Mobile Systems, Applications, and Services, New York, NY, USA, 2013, pp. 207-220.

[10] L. M. Ni, Y. Liu, Y. C. Lau, and A. P. Patil, "LANDMARC: indoor location sensing using active RFID," in Proceedings of the First IEEE International Conference on Pervasive Computing and Communications, 2003. (PerCom 2003)., 2003, pp. 407-415.

[11] J. Wang and D. Katabi, "Dude, Where's My Card?: RFID Positioning That Works with Multipath and Non-line of Sight," in Proceedings of the ACM SIGCOMM 2013 Conference on SIGCOMM, New York, NY, USA, 2013, pp. 51-62.

[12] L. Yang, Y. Chen, X.-Y. Li, C. Xiao, M. Li, and Y. Liu, "Tagoram: Real-time Tracking of Mobile RFID Tags to High Precision Using COTS Devices," in Proceedings of the 20th Annual International Conference on Mobile Computing and Networking, New York, NY, USA, 2014, pp. 237-248.

[13] W. Zhuo, B. Zhang, S. H. G. Chan, and E. Y. Chang, "Error Modeling and Estimation Fusion for Indoor Localization," in 2012 IEEE International Conference on Multimedia and Expo, 2012, pp. 741-746.

[14] Z. Sun, A. Purohit, K. Chen, S. Pan, T. Pering, and P. Zhang, "PANDAA: a physical arrangement detection technique for networked devices through ambient-sound awareness," in Proc. ACM UbiComp, 2011, pp. 425-434.
[15] W. Huang et al., "Shake and walk: Acoustic direction finding and fine-grained indoor localization using smartphones," in IEEE INFOCOM 2014 - IEEE Conference on Computer Communications, 2014, pp. 370-378.

[16] Y.-S. Kuo, P. Pannuto, K.-J. Hsiao, and P. Dutta, "Luxapose: Indoor Positioning with Mobile Phones and Visible Light," in Proceedings of the 20th Annual International Conference on Mobile Computing and Networking, New York, NY, USA, 2014, pp. 447-458.

[17] Z. Wang, Z. Yang, J. Zhang, C. Huang, and Q. Zhang, "Wearables Can Afford: Light-weight Indoor Positioning with Visible Light (Best Paper Candidate, Best Video Presentation Award)," Microsoft Res., May 2015.

[18] J. Chung, M. Donahoe, C. Schmandt, I.-J. Kim, P. Razavai, and M. Wiseman, "Indoor Location Sensing Using Geo-magnetism," in Proceedings of the 9th International Conference on Mobile Systems, Applications, and Services, New York, NY, USA, 2011, pp. 141154.

[19] H. Xie, T. Gu, X. Tao, H. Ye, and J. Lv, "MaLoc: A Practical Magnetic Fingerprinting Approach to Indoor Localization Using Smartphones," in Proceedings of the 2014 ACM International Joint Conference on Pervasive and Ubiquitous Computing, New York, NY, USA, 2014, pp. 243-253.

[20] J. Poushter, "Smartphone Ownership and Internet Usage Continues to Climb in Emerging Economies," Pew Research Center's Global Attitudes Project, 22-Feb-2016. .

[21] C. Gentile, N. Alsindi, R. Raulefs, and C. Teolis, Geolocation Techniques: Principles and Applications. Springer Science \& Business Media, 2012.

[22] S. He, W. Lin, and S. H. G. Chan, "Indoor Localization and Automatic Fingerprint Update with Altered AP Signals," IEEE Trans. Mob. Comput., vol. 16, no. 7, pp. 1897-1910, Jul. 2017.

[23] E. Laitinen and E. S. Lohan, "On the Choice of Access Point Selection Criterion and Other Position Estimation Characteristics for WLAN-Based Indoor Positioning," Sensors, vol. 16, no. 5, May 2016.

[24] S. Eisa, J. Peixoto, F. Meneses, and A. Moreira, "Removing useless APs and fingerprints from WiFi indoor positioning radio maps," in International Conference on Indoor Positioning and Indoor Navigation, 2013, pp. 1-7.

[25] S. Meyer, T. Vaupel, and S. Haimerl, "Wi-Fi coverage and propagation for localization purposes in permanently changing urban areas," in IADIS Multi Conference on Computer Science and Information Systems, MCCSIS 2008, 2008, pp. 11-20.

[26] T. Vaupel, J. Seitz, F. Kiefer, S. Haimerl, and J. Thielecke, "Wi-Fi positioning: System considerations and device calibration," in 2010 International Conference on Indoor Positioning and Indoor Navigation, 2010, pp. 1-7.

[27] "Correlation coefficients - MATLAB corrcoef." [Online]. Available:

https://www.mathworks.com/help/matlab/ref/corrcoef.html.

[Accessed: 08-May-2018].

[28] A. Gelman, J. B. Carlin, H. S. Stern, D. B. Dunson, A. Vehtari, and D. B. Rubin, Bayesian Data Analysis, Third Edition. CRC Press, 2013.

[29] "MLE vs MAP: the connection between Maximum Likelihood and Maximum A Posteriori Estimation - Agustinus Kristiadi's Blog." [Online]. Available: http://wiseodd.github.io/techblog/2017/01/01/mle-vs-map/. [Accessed: 12-Aug-2018].

[30] H. Rajaguru and S. K. Prabhakar, KNN Classifier and K-Means Clustering for Robust Classification of Epilepsy from EEG Signals. A Detailed Analysis. diplom.de, 2017. 